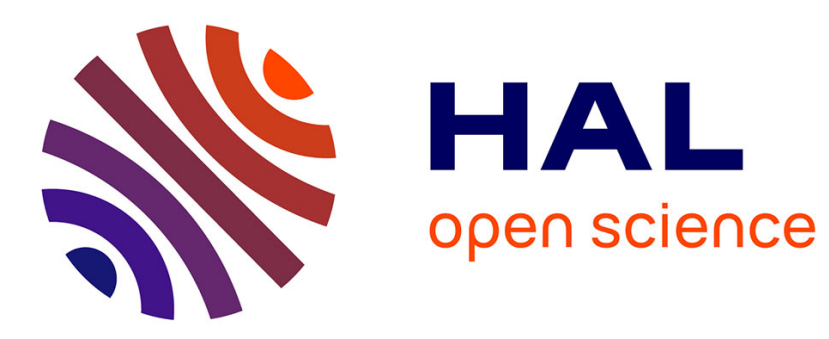

\title{
Data reduction for the AMBER instrument
}

Florentin Millour, E. Tatulli, A. Chelli, G. Duvert, G. Zins, B. Acke, F. Malbet

\section{To cite this version:}

Florentin Millour, E. Tatulli, A. Chelli, G. Duvert, G. Zins, et al.. Data reduction for the AMBER instrument. New Frontiers in Stellar Interferometry, 2004, France. pp.1222. hal-00008536

\section{HAL Id: hal-00008536 https://hal.science/hal-00008536}

Submitted on 7 Sep 2005

HAL is a multi-disciplinary open access archive for the deposit and dissemination of scientific research documents, whether they are published or not. The documents may come from teaching and research institutions in France or abroad, or from public or private research centers.
L'archive ouverte pluridisciplinaire HAL, est destinée au dépôt et à la diffusion de documents scientifiques de niveau recherche, publiés ou non, émanant des établissements d'enseignement et de recherche français ou étrangers, des laboratoires publics ou privés. 


\title{
Data reduction for the AMBER instrument
}

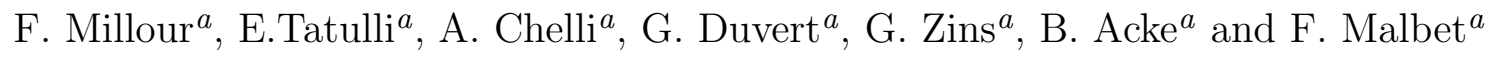 \\ ${ }^{a}$ LAOG, Observatoire de Grenoble, BP 53, F-38041 Grenoble CEDEX 9, FRANCE;
}

\begin{abstract}
We present here the general formalism and data processing steps used in the data reduction pipeline of the AMBER instrument. AMBER is a three-telescope interferometric beam combiner in $\mathrm{J}, \mathrm{H}$ and $\mathrm{K}$ bands installed at ESO's Very Large Telescope Interferometer. The fringes obtained on the 3 pairs of telescopes are spatially coded and spectrally dispersed. These are monitored on a 512x512 infrared camera at frame rates up to 100 frames per second, and this paper presents the algorithm used to retrieve the complex coherent visibility of the science target and the subsequent squared visibility, differential phase and phase closure on the 3 bases and in the 3 spectral bands available in AMBER.
\end{abstract}

Keywords: AMBER, VLTI, Long Baseline Infrared Interferometry, Complex Visibility, P2VM Algorithm, Data Reduction Software, Spectroscopy, Spatial Coding, Phase Closure, Interspectrum

\section{INTRODUCTION}

AMBER $^{1}$ makes fringes on an infrared detector. Before combination, a spatial filtering ${ }^{2}$ is made by optics fibers in order to keep only the central part of the airy disk of the telescope. These fringes are spatially coded, i.e. their spatial frequency is fixed by the instrument setup, and the spatial frequency of fringes due to a pair of telescopes is different from the spatial frequency of another pair. In the following we will treat the case of only one pair of telescopes. The case of 3 telescopes can easily be deduced from the equations given in this article.

The equations are given for one wavelength, so every value in this article should be interpreted as wavelength dependant. The interferometric equation describes the interferometric signal pixel per pixel:

$$
i_{k}=N p_{1} a_{1 k}+N p_{2} a_{2 k}+2 N V \sqrt{p_{1} p_{2}} \sqrt{a_{1 k} a_{2 k}} \cos \left(2 \pi f \alpha_{k}+\phi_{a k}+\phi_{p}+\phi_{o}\right)
$$

In this equation $k$ is the pixel index, $i_{k}$ is the number of photo-events in pixel $k$ of the interferometric channel, $N$ is the unknown object's flux, $p_{1}$ and $p_{2}$ are transmission coefficients for the two combined beams, and $a_{1 k}$ and $a_{2 k}$ are related to specific features of each pixel. $V$ is the amplitude of the complex visibility.

Furthermore, 4 phases are to be taken into account: $f$ corresponds to the combining baseline $b(f=b / \lambda), \alpha_{k}$ is an angle that indicates the position of each pixel, $\phi_{a k}$ is a phase factor that accounts for optical aberrations in the instrument (which are expected to be negligable) and $\phi_{o}$ is the object's phase. When measuring a source through the turbulent atmosphere, another phase factor enters the equation: the differential piston $\phi_{p}$. While measuring this interferogram, the photometric variability in both input beams is recorded simultaneously in the photometric channels. Let's call $P_{1}$ and $P_{2}$ the measured flux in photometric channels 1 and 2 respectively. Hence one can define the coefficients $v_{1 k}$ and $v_{2 k}$ as

$$
\begin{aligned}
& P_{1} v_{1 k}=N p_{1} a_{1 k} \\
& P_{2} v_{2 k}=N p_{2} a_{2 k}
\end{aligned}
$$

If one can determine the $v_{k}$ values, one can compute the continuum corrected interferogram $m_{k}$ defined by

$$
m_{k}=i_{k}-P_{1} v_{1 k}-P_{2} v_{2 k}=2 N V \sqrt{p_{1} p_{2}} \sqrt{a_{1 k} a_{2 k}} \cos \left(2 \pi f \alpha_{k}+\phi_{a k}+\phi_{p}+\phi_{o}\right)
$$

Notice that there are 3 sets of $v_{k}$ when one works with 3 input beams.

Further author information: (Send correspondence to F.M.)

F.M: E-mail: Florentin.Millour@obs.ujf-grenoble.fr, Telephone: +33 (0)476 635509 
In the case of AMBER data reduction, we decided not to use the "traditional" Fourier transform algorithm ${ }^{3}$ because of the precision required in the specifications of the instrument (1\% for the fringe contrast). For that purpose we developped an algorithm that generalizes to more than 4 pixels the well known ABCD algorithm and which is called "P2VM" ${ }^{4}$ as for Pixel-To-Visibility-Matrix algorithm. ${ }^{5}$ It takes into account the shape of the output beam of the instrument ${ }^{6}$ in order to accurately calibrate the intrumental factor in the visibilities and can be interpreted in the Fourier space as a Fourier transform where the shape of the fringe peak is fitted.

\section{THE DATA REDUCTION SOFTWARE}

\subsection{Overview}

For the implementation in the Data Reduction Software, we wrote a c library called amdlib (stands for AMber Dcs LIBrary) that handles the raw data, the calibration data and computes the P2VM and the subsequent visibilities $^{7}$ (figure 1). We decided to use the same library for quickLook on the AMBER workstation to help the astronomer to evaluate in real time the quality of the data, and for offline data reduction.

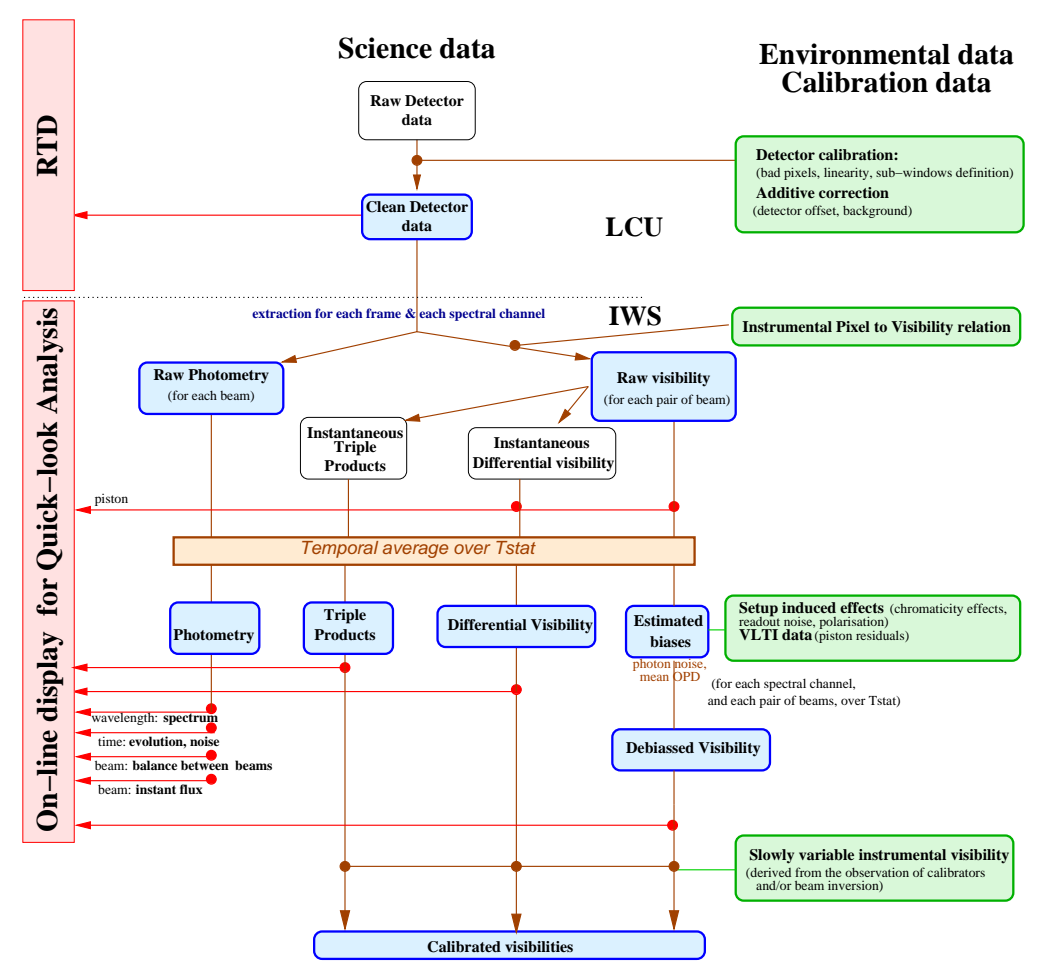

Figure 1. AMBER Data Reduction Roadmap

This library can be linked with a data reduction software like IDL, SciLab, etc. We developped at LAOG the complete linking with the open source IDL-like software of John Munro called Yorick (ftp://ftp-icf.llnl.gov/pub/Yorick/doc/index.html). The following figures illustrating this paper have been prepared with Yorick using amdlib on real fringes obtained on Sirius in March 2004 with VLTI-AMBER. ${ }^{8}$

The detector of AMBER takes only a part of the total detector array for rapid frames observing needs, as shown in figure 2. This is why the AMBER images seem to have sometimes "steep edges", when the detector windows are not well centered on the actual beam. 

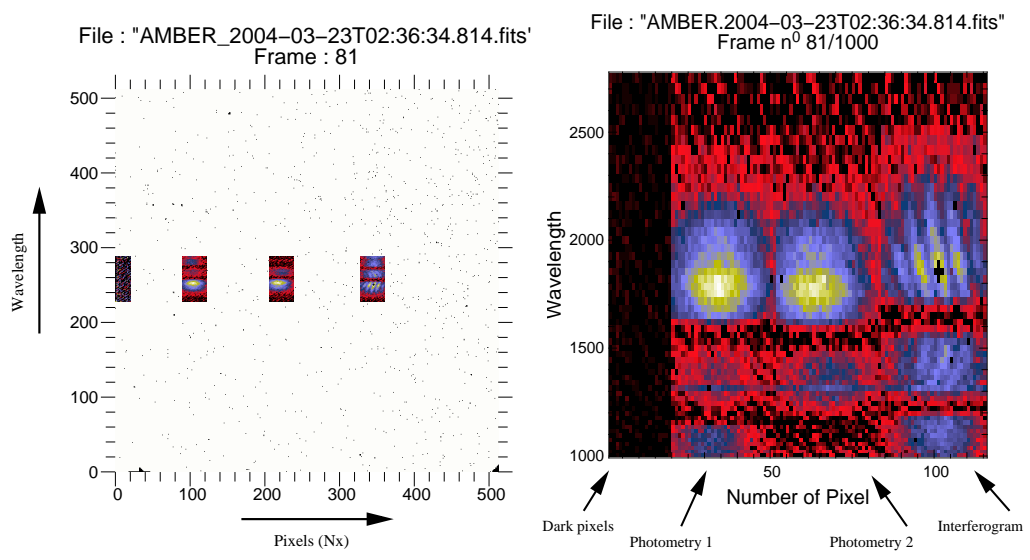

Figure 2. Placement of the data window in the detector array and a corresponding displayed frame in the data reduction software. Note that the wavelength table is not correct but it will be updated in the later steps of the data reduction.

\subsection{P2VM Computation Preparation}

For an accurate estimation of the complex visibilities of the scientific object, one needs to completely calibrate the behaviour of the instrument, which leads to accurately characterize the spatial frequency of the fringes and the shape of the output beams. The P2VM calibration is aimed at reaching this goal with some constraints as, for example, a very hard stability requirement of the instrument.

A P2VM "calibration" is performed by obtaining one frame with all shutters closed to get a 'sky-type' frame, then by opening in turn only one shutter to get the shape of the illumination in the interferometric channel due to one fiber (the so-called $v_{k}^{i}$ ), then by opening in turn pairs of shutters to retrieve the interference fringe pattern for each pair of telescope, with and wihout a phase shift of a known value $\gamma_{0}$ (figure 3 ).

The visibility of the artificial source (lamp) in the Calibration and Alignment Unit (CAU) of AMBER is supposed to be fixed, calibrated and noted $V_{c}$.

Those calibration frames are processed with amdlib routines to produce a P2VM used for all following visibility extractions, until the Instrument Setup changes and a new P2VM is needed.

\subsection{P2VM Computation}

The first of the previously described calibration frames is a sky-like measurement, without input light. This is done in order to substract the uncorrelated light 'sky' due to the thermal emission of the fibers, if any. The frames number 2 and 3 are exposures with only one input beam (figure 4 ). This allows one to compute the $v_{k}$ coefficients in the equations described before, by dividing the interferometric channel by the measured photometric flux, pixel per pixel and for every input beam.

The other 2 frames completely determine the calibration of the instrument. In these frames, two input beams at a time are combined. Two exposures are made per baseline. In one of this couple of frames, an additional phase shift is inserted into one of the beams. This results in an extra phase factor in the interferometric equation, which is called $\gamma_{0}$. Hence one has the following equations:

$$
i_{k}^{0}=N^{0} p_{1} a_{1 k}+N^{0} p_{2} a_{2 k}+2 N^{0} V_{c} \sqrt{p_{1} p_{2}} \sqrt{a_{1 k} a_{2 k}} \cos \left(2 \pi f \alpha_{k}+\phi_{a k}+\phi_{c}\right)
$$

for the frame without an additional phase shift (indicated as ${ }^{0}$ ) and

$$
i_{k}^{\gamma_{0}}=N^{\gamma_{0}} p_{1} a_{1 k}+N^{\gamma_{0}} p_{2} a_{2 k}+2 N^{\gamma_{0}} V_{c} \sqrt{p_{1} p_{2}} \sqrt{a_{1 k} a_{2 k}} \cos \left(2 \pi f \alpha_{k}+\phi_{a k}+\phi_{c}+\gamma_{0}\right)
$$

for the frame with an extra phase shift (indicated as $\gamma_{0}$ ). $V_{c}$ and $\phi_{c}$ are respectively the known visibility and phase of the calibration source. The continuum corrected interferograms for both frames are

$$
\begin{array}{r}
m_{k}^{0}=2 N^{0} V_{c} \sqrt{p_{1} p_{2}} \sqrt{a_{1 k} a_{2 k}} \cos \left(2 \pi f \alpha_{k}+\phi_{a k}+\phi_{c}\right) \\
m_{k}^{\gamma_{0}}=2 N^{\gamma_{0}} V_{c} \sqrt{p_{1} p_{2}} \sqrt{a_{1 k} a_{2 k}} \cos \left(2 \pi f \alpha_{k}+\phi_{a k}+\phi_{c}+\gamma_{0}\right)
\end{array}
$$




\begin{tabular}{|c|c|c|c|c|}
\hline Shutter 1 & Shutter 2 & Delaying plate & file Type & Image \\
\hline Close & Close & No Delay & Dark & \\
\hline Open & Close & No Delay & Photometric & \\
\hline Close & Open & No Delay & Photometric & \\
\hline Open & Open & No Delay & Interferometric & \\
\hline Open & Open & 1/2 Delayed & Interferometric & \\
\hline
\end{tabular}

Figure 3. Complete calibration sequence for 2 telescopes taken with the internal calibration source (CAU)
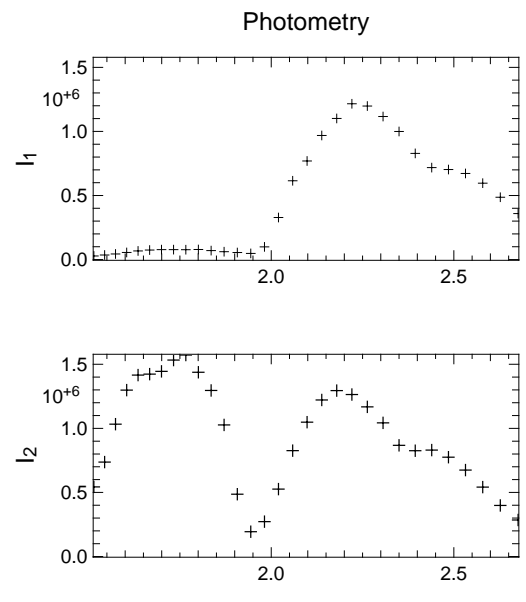

Wavelenght $(\mu \mathrm{m})$

Figure 4. Photometry extraction from calibration files 
Knowing of $P_{1}^{0} v_{1 k}, P_{2}^{0} v_{2 k}, P_{1}^{\gamma_{0}} v_{1 k}$ and $P_{2}^{\gamma_{0}} v_{2 k}$ and the relations 2 and 3 are used to eliminate the unknown fluxes $N^{0}$ and $N^{\gamma_{0}}$ from equations 7 and 8. One obtains

$$
\begin{aligned}
m_{k}^{0} & =2 V_{c} \sqrt{\sum_{j=1}^{N_{x}} P_{1}^{0} P_{2}^{0} v_{1 j} v_{2 j}} \sqrt{\frac{a_{1 k} a_{2 k}}{\sum_{j=1}^{N x} a_{1 j} a_{2 j}}} \cos \left(2 \pi f \alpha_{k}+\phi_{a k}+\phi_{c}\right) \\
m_{k}^{\gamma_{0}} & =2 V_{c} \sqrt{\sum_{j=1}^{N_{x}} P_{1}^{\gamma_{0}} P_{2}^{\gamma_{0}} v_{1 j} v_{2 j} \sqrt{\frac{a_{1 k} a_{2 k}}{\sum_{j=1}^{N x} a_{1 j} a_{2 j}}}} \cos \left(2 \pi f \alpha_{k}+\phi_{a k}+\phi_{c}+\gamma_{0}\right)
\end{aligned}
$$

$N_{x}$ is the number of pixels in the spatial direction per interferogram.

\subsection{Obtention of the Carrying Wave (P2VM)}

At this point, one can define the coefficients $c_{k}$ and $d_{k}$,

$$
\begin{gathered}
c_{k}=\sqrt{\frac{a_{1 k} a_{2 k}}{\sum_{j=1}^{N x} a_{1 j} a_{2 j}}} \cos \left(2 \pi f \alpha_{k}+\phi_{a k}+\phi_{c}\right) \\
d_{k}=\sqrt{\frac{a_{1 k} a_{2 k}}{\sum_{j=1}^{N x} a_{1 j} a_{2 j}}} \sin \left(2 \pi f \alpha_{k}+\phi_{a k}+\phi_{c}\right)
\end{gathered}
$$

called the real and imaginary part of the carrying wave of the interferometer (for this wavelength and for this baseline). Making use of this notation, one can write the continuum corrected interferograms as:

$$
\begin{aligned}
m_{k}^{0} & =2 V_{c} \sqrt{\sum_{j=1}^{N_{x}} P_{1}^{0} P_{2}^{0} v_{1 j} v_{2 j}} \times c_{k} \\
m_{k}^{\gamma_{0}} & =2 V_{c} \sqrt{\sum_{j=1}^{N_{x}} P_{1}^{\gamma_{0}} P_{2}^{\gamma_{0}} v_{1 j} v_{2 j}} \times\left(c_{k} \cos \gamma_{0}-d_{k} \sin \gamma_{0}\right)
\end{aligned}
$$

In these equations, $c_{k}$ and $d_{k}$ are the only unknown values, so they can be computed.
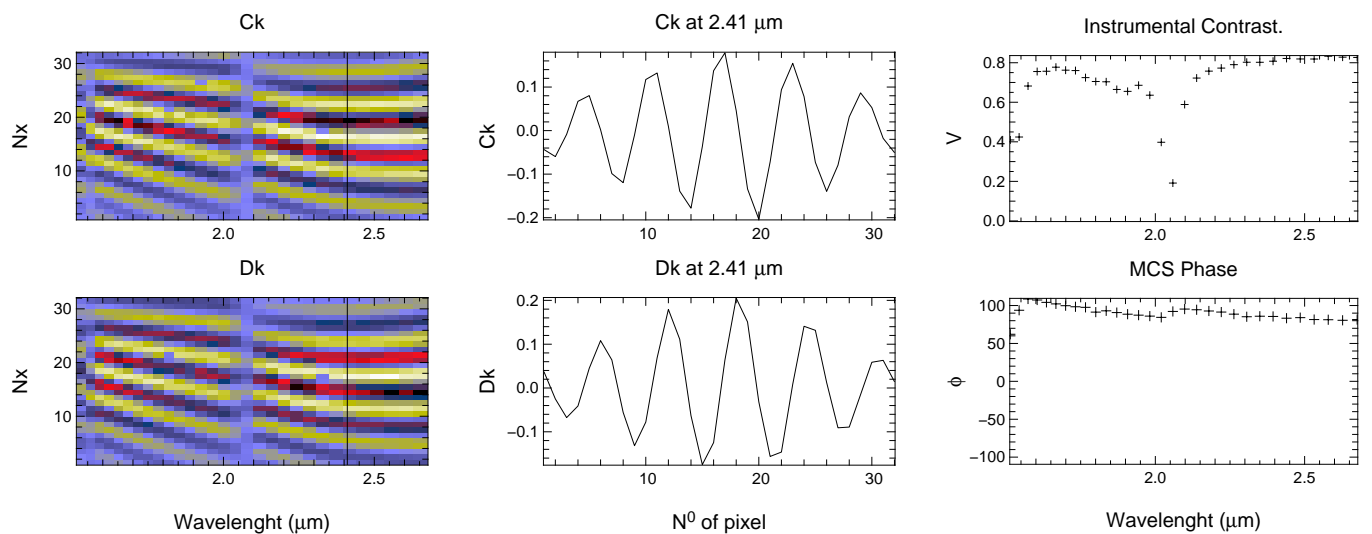

Figure 5. The carrying waves obtained in the P2VM in the 2 telescopes case (left \& middle), the corresponding instrumental contrast (top right) and the phase $\gamma_{0}$ that can be recalibrated from the P2VM data (bottom right)

The real and imaginary parts of the carrying waves for each pixel and for all wavelengths are the inputs of the pixel-to-visibility matrix P2VM (figure 5). Thus, this matrix has dimensions $\left(N_{x} \times 6\right)$ in the case of 3 telescopes and $\left(N_{x} \times 2\right)$ in the case of 2 telescopes for each wavelength. The computed $v_{k}$ coefficients are also saved and 
provided to the second part of the data reduction. One can determine the instrumental contrast by the data contained in the P2VM (figure 5):

$$
V_{i n s t}^{2}=\sum_{j=1}^{N x} c_{k}^{2}+d_{k}^{2}
$$

\subsection{Visibility extraction}

Once a P2VM has been recorded, visibility extraction can be performed on all following observations. Indeed, let us start with the interferometric equation for two telescopes:

$$
i_{k}=N p_{1} a_{1 k}+N p_{2} a_{2 k}+2 N V_{(12)} \sqrt{p_{1} p_{2}} \sqrt{a_{1 k} a_{2 k}} \cos \left(2 \pi f_{(12)} \alpha_{k}+\phi_{a k}^{(12)}+\phi_{p}^{(12)}+\phi_{o}^{(12)}\right)
$$

One can remark that the spatial coding of the interferogram is captured in the quantity $f_{(12)}$. One can use the monitored photometric flux $P_{1} v_{1 k}, P_{2} v_{2 k}$ and the calibrated $v_{i k}$ coefficients in each beam to pass from the interferogram given by equation 16 to the continuum corrected interferogram with the relations 2 and 3 . Let us consider the continuum corrected interferogram for the two telescopes case.

$$
m_{k}=2 N V_{(12)} \sqrt{p_{1} p_{2}} \sqrt{a_{1 k} a_{2 k}} \cos \left(2 \pi f_{(12)} \alpha_{k}^{(12)}+\phi_{a k}^{(12)}+\phi_{p}^{(12)}+\phi_{o}^{(12)}+\phi_{c}^{(12)}-\phi_{c}^{(12)}\right)
$$

Defining $\Phi^{(12)}=\phi_{p}^{(12)}+\phi_{o}^{(12)}-\phi_{c}^{(12)}$ for baseline 12 leads to

$$
m_{k}=2 N V_{(12)} \sqrt{p_{1} p_{2}} \sqrt{\sum_{j=1}^{N_{x}} a_{1 j} a_{2 j}}\left(c_{k}^{(12)} \cos \Phi^{(12)}-d_{k}^{(12)} \sin \Phi^{(12)}\right)
$$

One can define the weighted complex visibility for baseline 12 as $C_{12}=R_{12}+i I_{12}$ where

$$
R_{12}=2 N V_{(12)} \sqrt{p_{1} p_{2}} \sqrt{\sum_{j=1}^{N_{x}} a_{1 j} a_{2 j}} \times \cos \Phi^{(12)}
$$

and

$$
I_{12}=2 N V_{(12)} \sqrt{p_{1} p_{2}} \sqrt{\sum_{j=1}^{N_{x}} a_{1 j} a_{2 j}} \times \sin \Phi^{(12)}
$$

are the real and imaginary parts. From equations 18, 19 and 20 the meaning of the name 'carrying waves' is clear. They are the physical quantities that support the weighted complex visibilities.

The continuum corrected interferogram for two baselines (18) can now be written as:

$$
m_{k}=c_{k}^{(12)} R_{12}-d_{k}^{(12)} I_{12}
$$

The $R_{12}$ and $I_{12}$ are estimated from the data for every science frame and wavelength by minimising

$$
\chi_{R, I}^{2}=\sum_{j=1}^{N_{x}}\left(\frac{m_{j}-c_{j}^{(12)} R_{12}+d_{j}^{(12)} I_{12}}{\sigma_{m_{j}}}\right)^{2}
$$

In this formula, $\sigma_{m_{j}}^{2}$ is the formal variance of $m_{j}$ (assuming only photon and detector noises):

$$
\sigma_{m_{j}}^{2}=\sigma_{i_{j}}^{2}+\sum_{k=1}^{2} v_{k j}^{2} \sigma_{P_{k}}^{2}
$$

assuming Poisson statistics

$$
\begin{aligned}
\sigma_{i_{j}}^{2} & =\bar{i}_{j}+\sigma_{d e t}^{2} \\
\sigma_{P_{k}}^{2} & =\bar{P}_{k}+\sigma_{d e t}^{2} N_{x}
\end{aligned}
$$


$\sigma_{\text {det }}^{2}$ is the detector noise $\left(\approx 15 e^{-}\right)$. For fair enough signal-to-noise rates, $\sigma_{m_{j}}^{2}$ can be defined instantaneously, with $\overline{i_{j}}=i_{j}$ and $\bar{P}_{k}=P_{k}$. The atmospheric influence on this variance is neglected at this point.

Notice that one can write equation 21 as a matrix multiplication, where $\left[m_{k}\right], P 2 V M$ and $[R, I]$ respectively have dimensions $\left(N_{x} \times 1\right),\left(N_{x} \times 2\right)$ and $(2 \times 1)$ for each spectral channel.

$$
\left[m_{k}\right]=P 2 V M \times[R, I]
$$

Finding the linear least square fit values for $[R, I]$ is equivalent to 'invert' P2VM, taking into account the pixelto-pixel covariance matrix $C O V$.

$$
\begin{aligned}
{[R, I] } & =\left({ }^{t} P 2 V M \times C O V^{-1} \times P 2 V M\right)^{-1} \times{ }^{t} P 2 V M \times C O V^{-1}\left[m_{k}\right] \\
& =i n v P 2 V M\left[m_{k}\right]
\end{aligned}
$$

Notice that $\left({ }^{t} P 2 V M \times C O V^{-1} \times P 2 V M\right)$ is a square symmetrical matrix with non-zero diagonal entries, so inverting is possible. The covariance matrix is a $\left(N_{x} \times N_{x}\right)$ diagonal matrix with

$$
C O V(j, j)=\sigma_{m_{j}}^{2}
$$

for $j=1 \ldots N_{x}$. The cross-correlation of two different pixels is neglected here. It is also possible to define the covariance matrix as an average over a certain number of frames, using equation 23 with averaged values $\overline{i_{j}}=<i_{j}>_{\text {frames }}$ and $\bar{P}_{k}=<P_{k}>_{\text {frames. }}$. This avoids numerous inversions (for each frame) during the calculations of the $R$ and $I$ quantities.

The figure 6 shows the phases (arcTangent of $I / R$ ) of the complex visibilities computed in amdlib. The wavelength table was not up to date at the time of the observation but the phases are well aligned in the $\mathrm{K}$ band (where there is enough flux).

\subsection{From weighted complex visibilities to visibilities}

The weighted complex visibilities are the bricks to build the required observables (squared visibility, differential phase, closure phase, etc.). We will present here only the squared visibility estimation.
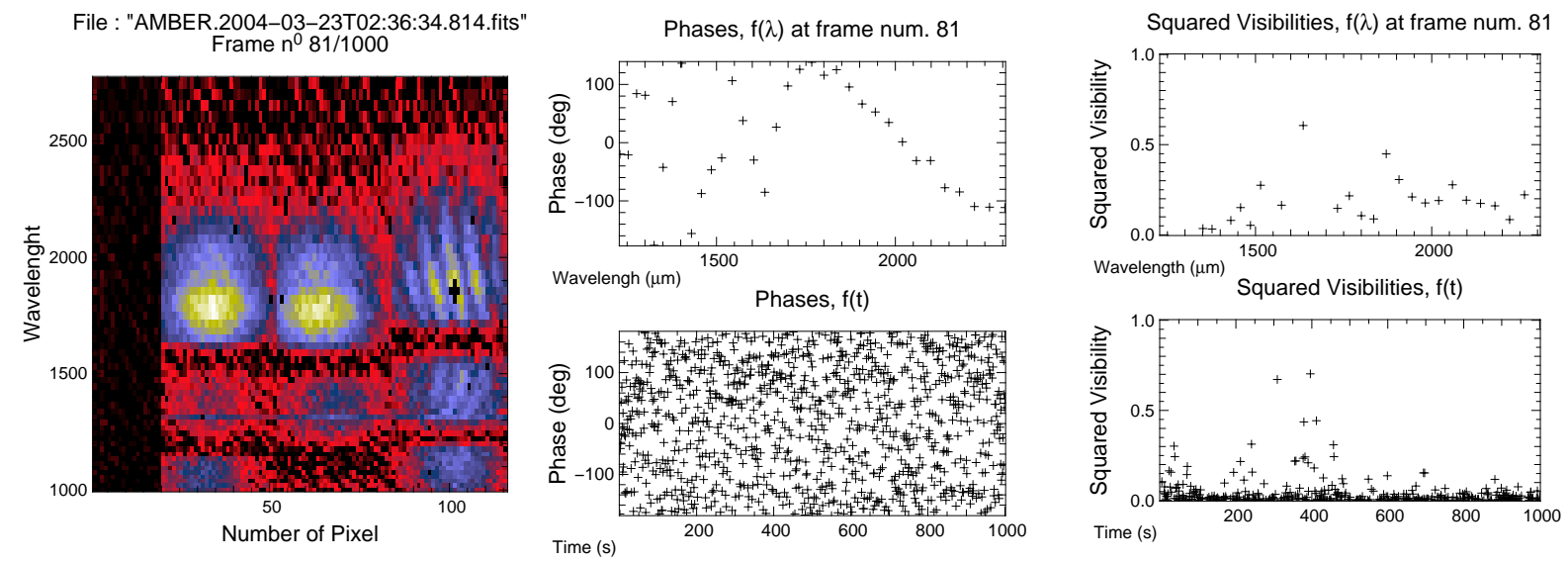

Figure 6. Example of an observation taken on Sirius during Assembly, Integration and Verification of $\mathrm{AMBER}^{9}$ in $\mathrm{March}$ 2004 (fringe pattern at the left). Top middle is the instantaneous visibilities phase on a frame where there was flux in both telescopes. The $\mathrm{H}$ band did not have much flux, that is why the phases seems erratic while in the $\mathrm{K}$ band there was flux and the phases are well aligned. This was not all the time the case and is why the phase in one spectral channel is uniformely spanned (bottom middle). In the same frame, the instantaneous squared visibilities are quite high (top right) as in one spectral channel they are almost always null as the photometry is low (bottom right) due to the bad injection in the fibers with the testing siderostat of the VLTI. 
The visibility is estimated starting from the squared norm of $C_{12}$, averaged over a number of frames. Again we use formulas 2 and 3 to estimate the unknown $p_{r}$ and $a_{r k}$ values. Inserting these expressions into equations 19 and 20, one obtains with $\left|C_{12}\right|^{2}-\operatorname{bias}(t)=R_{12}^{2}+I_{12}^{2}-\operatorname{bias}(t)$

$$
V_{12}^{2}=\frac{<R_{12}^{2}+I_{12}^{2}-\operatorname{bias}(t)>_{t}}{4<P_{1} P_{2}>_{t} \sum_{j=1}^{N_{x}} v_{1 j} v_{2 j}}
$$

The unbiasing of the squared weighted visibilities is necessary, as for the Fourier transform algorithm. ${ }^{10}$ This leads to a change on the error on $C_{12}$ with an expected value of 0 into a squared error with a non-zero mean. The debiasing is done instantaneously by estimating the photon bias and the detector bias.

The figure 6 shows an instantaneous measurement of the squared visibility on the star Sirius taken during April 2004 at Paranal. The measured precision on the sky is about 1\% so the goal of getting accurate visibilities with the P2VM has been achieved in the case of AMBER.

\subsection{The differential phase estimator}

The unbiased differential phase between $\lambda_{1}$ and $\lambda_{2}$ is given directly from the averaged interspectrum of the complex visibility $C_{12}$ by

$$
\Delta \Phi_{\lambda_{1} \lambda_{2}}=\text { atan }<C_{12, \lambda_{1}} C_{12, \lambda_{2}}^{*}>
$$

\subsection{The closure phase estimator}

As for the differential phase, the closure phase is given directly from the averaged triple product of the complex visibilities $C_{12} C_{23}$ and $C_{13}$, only in the 3 telescopes case by

$$
\Phi_{123}^{\text {object }}=\text { atan }<C_{12} C_{23} C_{13}^{*}>+\Phi_{123}^{\text {instrument }}
$$

where $\Phi_{123}^{\text {instrument }}$ has to be calibrated on a point source.

\section{CONCLUSION}

In this paper we present the AMBER data reduction software based on a generalization of the ABCD method to get visibilities called "Pixel To Visibilities Matrix" algorithm. With this formalism, one can accurately calibrate the instrument configuration and get accurate measurements of the source visibilities, closure phases and differential phases.

One can then use these observables, corrected from the atmosphere transfert function ${ }^{11}$ to extract the useful physical parameters of the observed scientific object. 


\section{REFERENCES}

1. R. G. Petrov, F. Malbet, G. Weigelt, F. Lisi, P. Puget, P. Antonelli, U. Beckmann, S. Lagarde, E. Lecoarer, S. Robbe-Dubois, G. Duvert, S. Gennari, A. Chelli, M. Dugue, K. Rousselet-Perraut, M. Vannier, and D. Mourard, "Using the near infrared VLTI instrument AMBER," in Interferometry for Optical Astronomy II. Edited by Wesley A. Traub . Proceedings of the SPIE, Volume 4838, pp. 924-933, Feb. 2003.

2. P. Mege, F. Malbet, and A. Chelli, "Spatial filtering in AMBER," in Proc. SPIE Vol. 4006, p. 299-307, Interferometry in Optical Astronomy, Pierre J. Lena; Andreas Quirrenbach; Eds., pp. 299-307, July 2000.

3. V. Coudé Du Foresto, S. Ridgway, and J.-M. Mariotti, "Deriving object visibilities from interferograms obtained with a fiber stellar interferometer," A\&SAS 121, pp. 379-392, Feb. 1997.

4. K. H. Hofmann, MEMO AMBER-IGR-013 : Calibrating the visibilities, Dec. 1999.

5. A. Chelli, MEMO AMBER-IGR-018 : Visibility, Differential Phase and Closure Phase Estimators in the Image Space, July 2000.

6. K. H. Hofmann and F. Malbet, MEMO AMBER-OSM-006: A proposition for the AMBER visibility estimator, May 1998.

7. G. Duvert, R. G. Petrov, and P. Antonelli, VLT-PLA-AMB-15830-6004: AMBER Data Reduction Plan, Oct. 2003.

8. ESO Press Release, "Adding New Colours to Interferometry," Apr. 2004.

9. S. Robbe-Dubois, R. G. Petrov, U. Beckmann, S. Lagarde, F. Lisi, F. Malbet, P. Antonelli, Y. Bresson, A. Roussel, D. Mourard, A. Delboulbe, G. Duvert, P. Kern, E. LeCoarer, F. Millour, K. Rousselet-Perraut, G. Zins, M. Heininger, G. Weigelt, P. Stefanini, M. Accardo, C. Gil, N. Haddad, N. Housen, M. Kiekebusch, P. Mardones, F. Puech, F. Rantakyro, A. Richichi, M. Schoeller, and M. Vannier, "The VLTI focal instrument Amber : results of the first phase of the Alignment, Integration and Verification in Paranal," in Astronomical Telescopes and Instrumentation, New frontiers in Stellar Interferometry, Proceedings of the SPIE, Volume 5491: these proceedings, July 2004.

10. G. Perrin, "Subtracting the photon noise bias from single-mode optical interferometer visibilities," $A \mathscr{E} A$ 398, pp. 385-390, Jan. 2003.

11. G. Perrin, "The calibration of interferometric visibilities obtained with single-mode optical interferometers. Computation of error bars and correlations," A $\mathscr{E} A$ 400, pp. 1173-1181, Mar. 2003. 